\title{
Afrontamiento en pacientes con diabetes mellitus 2 con y sin retinopatía
}

\section{Coping in type 2 diabetes mellitus patients with and without retinopathy}

\author{
Francisco Javier Rosas-Santiago ${ }^{l}$, Ana Delia López-Suárez ${ }^{1}$ \\ y Samantha Jiménez-Herrera ${ }^{2}$
}

Citación: Rosas-Santiago, F.J., López-Suárez, A.D. y Jiménez-Herrera, S. (2019). Afrontamiento en pacientes con diabetes mellitus 2 con y sin retinopatía. Psicología y Salud, 29(2), 219-224.

\section{RESUMEN}

\begin{abstract}
Aun cuando se sabe que la forma en que los pacientes con diabetes mellitus 2 hacen frente al estrés repercute en su salud, no se ha estudiado la relación entre el afrontamiento y la retinopatía diabética. Esta investigación, hecha con una muestra por conveniencia de 42 sujetos, tuvo como objetivo comparar mediante autoinforme las estrategias de afrontamiento del estrés reportadas por pacientes diabéticos con y sin retinopatía. En grupos equivalentes en tamaño, años de aparición de la enfermedad y otros datos generales, como sexo, edad, escolaridad y estado civil, se encontraron diferencias estadísticamente significativas en la estrategia de afrontamiento denominada expresión emocional, la cual empleaban con mayor frecuencia los pacientes sin retinopatía. Este importante hallazgo abre una ventana de oportunidad para seguir explorando el papel del afrontamiento en el curso y evolución de las complicaciones asociadas a la diabetes mellitus 2. Se discuten las implicaciones de los hallazgos para la práctica clínica.
\end{abstract}

Palabras clave: Estrategias de afrontamiento; Expresión emocional; Retinopatía diabética.

\begin{abstract}
There is currently little or no specific research on the relationship between coping and diabetic retinopathy, even though it is known that stress coping by these patients is associated to the course and evolution of the disease. The present study used a convenience sample of 42 participants with diabetes mellitus type 2, in order to compare the stress-coping skills of patients with and without diabetic retinopathy, through a self-report inventory. Groups were equivalent in size, years with the disease, and such characteristics, as sex, age, schooling, and marital status. Results revealed statistically significant differences in coping through emotional expression: patients without retinopathy used this strategy more frequently than those with it. This finding is important in the sense that it opens a new window of opportunity to explore the role of coping in the progression of type 2 diabetes and its complications. Some implications of these findings for clinical practice are discussed.
\end{abstract}

Key words: Coping skills; Emotional expression; Diabetic retinopathy.

\footnotetext{
${ }^{1}$ Instituto de Investigaciones Psicológicas de la Universidad Veracruzana. Av. Dr. Luis Castelazo Ayala s/n, Col. Industrial Ánimas, 91190 Xalapa, Ver., México, tel. (228)841-89-00, ext. 13219, correo electrónico: frrosas@uv.mx. Artículo recibido el 12 de junio y aceptado el 17 de septiembre de 2018.

${ }^{2}$ Universidad de Xalapa, Carr. Xalapa-Veracruz Km 2, Núm. 341, Col. Acueducto Ánimas, 91190 Xalapa, Ver., tel. (228)841-72-25.
} 


\section{INTRODUCCIÓN}

$\mathrm{L}$ a diabetes mellitus tipo 2 (DM2 en lo sucesivo) es un trastorno metabólico crónico que se caracteriza por concentraciones elevadas y persistentes de glucosa en la sangre, consecuencia de deficiencias o alteraciones en la acción de la insulina en el organismo (Escobar et al., 2000). La proporción de adultos mexicanos con diagnóstico médico de DM2 es de $9.2 \%$, por lo que se considera un problema de salud pública (Gutiérrez et al., 2012) que muy probablemente crecerá en las próximas décadas (Fernández, Martínez, Martínez, Benedi y Traver, 2015), lo que obliga a emprender diversas acciones para su prevención y atención desde la perspectiva de diversas disciplinas, como la medicina, la psicología y la sociología, entre otras.

El buen manejo de la DM2 consiste en mantener los niveles adecuados de glucosa en la sangre, mismos que se ven afectados por aspectos metabólicos que dependen en gran medida de los hábitos de vida saludable del paciente (Covarrubias, Delgado, Rojas y Coria, 2017; Fernandez et al., 2015), mismos que incluyen comportamientos tales como un ejercicio regular, alimentación adecuada y manejo efectivo del estrés (García, Rodríguez, Rentería, Rodríguez y Melchor, 2016). Cuando no se le controla, la DM2 afecta gravemente distintos sistemas del organismo, todos ellos asociadas al daño vascular. La retinopatía diabética (RD en adelante) es la complicación más frecuente en pacientes con DM2; se trata de una lesión oftalmológica prevenible que se estima como una de las principales causas de ceguera en esta población (Rodríguez, 2015).

La psicología desempeña un papel fundamental en la comprensión de comportamientos ligados al curso y evolución de la DM2, pues a partir del diagnóstico de la enfermedad las personas deben desarrollar comportamientos de autocuidado durante toda la vida, lo que desencadena un proceso de adaptación a las actividades de la vida diaria y la necesidad de tener información y conocimientos sobre el manejo de la enfermedad que les haga posible su control efectivo (Romero, Dos Santos, Martins y Zanetti, 2010).

Las estrategias de afrontamiento ante el estrés se hallan entre los factores psicológicos aso- ciados a la diabetes más estudiados (Hara et al., 2014), mismas que son definidas como los "procesos cognitivos y conductuales constantemente cambiantes que se desarrollan para manejar las demandas específicas externas o internas que son evaluadas como excedentes o desbordantes de los recursos del individuo" (Lazarus y Folkman, 1984, p. 141).

De manera general, tales estrategias se clasifican en dos grandes grupos: las activas, que consisten en un conjunto de conductas realizadas por el individuo con el propósito de modificar la situación estresante, y las pasivas, que consisten en acciones de regulación cognitiva y emocional cuyo fin es reducir el estrés percibido (Lazarus y Folkman, 1991).

En la población de pacientes con DM2 las estrategias de afrontamiento activo están relacionadas con una mejor regulación de los niveles de glucosa en sangre (Huang et al., 2016) y una adecuada adherencia al tratamiento médico (Azzollini, Bail, Vidal, Benvenuto y Ferrer, 2015). A su vez, las estrategias de afrontamiento pasivo se relacionan con menores índices de estrés asociado con la enfermedad (Yazla et al., 2018) y con menos problemas de convivencia social en los grupos de pertenencia (Nishio y Chujo, 2017); sin embargo, la relación que existe entre el afrontamiento y los daños al organismo asociados a la DM2 se ha explorado escasamente.

Dada la importancia de los factores comportamentales en el desarrollo de la RD en pacientes con DM2, se hace necesario explorar las formas en que el afrontamiento del estrés podría asociarse con dicha condición. Por ello, el propósito de esta investigación cuantitativa, transversal, no experimental y comparativa de dos grupos fue contrastar las estrategias de afrontamiento del estrés en pacientes con DM2 con y sin RD.

\section{MÉTODO}

\section{Participantes}

La muestra por conveniencia estuvo conformada por 42 pacientes de 30 a 80 años de edad $(\mathrm{M}=63.1$ \pm 8.9 ) con diagnóstico confirmado de DM2, sometidos a exploración oftalmológica el mismo día en 
que se aplicaron los instrumentos, que aceptaron participar en el estudio y que respondieron los cuestionarios. Esta muestra se dividió en dos grupos de 21 pacientes: con y sin RD. Para no afectar la variable de afrontamiento, se excluyó del estudio a aquellos que refirieron algún antecedente psiquiátrico y a quienes se encontraban en algún proceso de psicoterapia.

\section{Instrumentos}

Cuestionario elaborado ex profeso de datos generales, que incluyó la edad, el sexo, el estado civil, la escolaridad y el tiempo de padecer DM2.

Inventario de Estrategias de Afrontamiento (Cano, Rodríguez y García, 2007). Este instrumento consta de 40 reactivos que miden la frecuencia de uso de ocho estrategias de afrontamiento en una escala de 0 ("En absoluto") a 4 ("Totalmente"), en subescalas con cinco reactivos cada una (M teóri$\mathrm{ca}=10.0 \pm 1.0$ ) denominadas del modo siguiente: Resolución de problemas: estrategias cognitivas y conductuales encaminadas a eliminar el estrés modificando la situación que lo produce; Reestructuración cognitiva: estrategias cognitivas que modifican el significado de la situación estresante; Apoyo social: estrategias referidas a la búsqueda de apoyo emocional; Expresión emocional: estrategias para liberar las emociones que acontecen en el proceso de estrés; Evitación de problemas: estrategias que incluyen la negación y la evitación de pensamientos o actos relacionados con el acontecimiento estresante; Pensamiento desiderativo: estrategias cognitivas que reflejan el deseo de que la realidad no fuera estresante; Retirada social: estrategias que se emplean para alejarse de amigos, familiares, compañeros y personas significativas asociadas con la reacción emocional en el proceso estresante, y Autocrítica: estrategias basadas en culparse y criticarse a sí mismo por la ocurrencia de la situación estresante o por su manejo inadecuado.

En población mexicana, la confiabilidad de todas las subescalas fluctuó entre .70 y .86, siendo de .81 la de escala general (Quiroz, Méndez, Valero y Trujano, 2010).

\section{Procedimiento}

Una vez que las autoridades del Instituto de Seguridad y Servicios Sociales de los Trabajadores del Estado (ISSSTE) de la ciudad de Xalapa (México) evaluaron el proyecto de investigación, autorizaron su desarrollo en el módulo de atención del Programa de Manejo Integral de Diabetes por Etapas, que brinda atención multidisciplinaria para evitar el desarrollo de complicaciones asociadas con la diabetes, incluida la RD.

La conformación de la muestra se llevó a cabo una vez que el personal médico realizaba la exploración oftalmológica de rutina a los pacientes como parte de la valoración médica integral. Dicho personal informaba a los pacientes si sufrían o no alguna complicación en la retina, tras de los cual los invitaban a acudir al consultorio de atención psicológica del módulo. Una vez allí, se les invitaba a participar en el estudio. Quienes aceptaban, firmaban un formato de consentimiento informado en el que indicaban si habían recibido o no un diagnóstico de RD, tras de lo cual dicho diagnóstico era corroborado por el médico a cargo.

La aplicación de los instrumentos se llevó a cabo de manera individual, y un psicólogo del equipo de investigación auxilió a los participantes para su correcto llenado. Los datos fueron colectados a lo largo de cinco meses. Los pacientes que participaron recibieron los resultados de la evaluación y asesoría psicológica a quienes la solicitaron.

\section{Análisis de datos}

Se llevó a cabo un análisis descriptivo de los datos sociodemográficos y psicológicos a través del cálculo de frecuencias, medias y desviaciones estándar. Para la estadística inferencial, se hicieron pruebas de normalidad de Kolmogorov-Smirnov, y para comparar las puntuaciones de las estrategias de afrontamiento entre ambos grupos se utilizó estadística no paramétrica con la prueba U de Mann-Whitney y la chi cuadrada, empleando para ello el software SPSS, v. 13.0. El nivel de confianza obtenido fue de .95 en todos los casos. 


\section{RESULTADOS}

Se realizó la prueba de normalidad para las variables numéricas; al hallarse que no se distribuían normalmente, se emplearon las técnicas no paramétricas. Se compararon los grupos en cuanto a sus características generales, comprobándose su equivalencia, tal como se muestra en la Tabla 1.

Tabla 1. Comparación de características sociodemográficas y tiempo de padecimiento.

\begin{tabular}{|c|c|c|c|c|c|}
\hline \multirow[t]{2}{*}{ CARACTERÍSTICAS } & \multicolumn{2}{|c|}{$\begin{array}{c}\text { Sin RD } \\
n=21\end{array}$} & \multicolumn{2}{|c|}{$\begin{array}{c}\text { Con RD } \\
n=21\end{array}$} & \multirow[t]{2}{*}{$p$} \\
\hline & M & D.E. & $\mathbf{M}$ & D.E. & \\
\hline Tiempo en años de padecer diabetes & 7.9 & 6.6 & 4.4 & 3.5 & \multirow{2}{*}{$\begin{array}{l}.08+ \\
.51+\end{array}$} \\
\hline Años de edad & 62.2 & 9.9 & 64.1 & 7.9 & \\
\hline \multicolumn{6}{|l|}{ Sexo } \\
\hline Hombres & \multicolumn{2}{|c|}{13} & \multicolumn{2}{|c|}{8} & \multirow{2}{*}{$.54++$} \\
\hline Mujeres & \multicolumn{2}{|c|}{10} & \multicolumn{2}{|c|}{11} & \\
\hline \multicolumn{6}{|l|}{ Estado civil } \\
\hline Solteros & \multicolumn{2}{|c|}{1} & \multicolumn{2}{|c|}{2} & \multirow{5}{*}{$.27++$} \\
\hline Unión libre & \multicolumn{2}{|c|}{4} & \multicolumn{2}{|c|}{3} & \\
\hline Casados & \multicolumn{2}{|c|}{10} & \multicolumn{2}{|c|}{15} & \\
\hline Divorciados & \multicolumn{2}{|c|}{1} & \multicolumn{2}{|c|}{0} & \\
\hline Viudos & \multicolumn{2}{|c|}{5} & \multicolumn{2}{|c|}{1} & \\
\hline \multicolumn{6}{|l|}{ Escolaridad } \\
\hline Primaria & \multicolumn{2}{|c|}{4} & \multicolumn{2}{|c|}{6} & \multirow{5}{*}{$.29++$} \\
\hline Secundaria & \multicolumn{2}{|c|}{4} & \multicolumn{2}{|c|}{5} & \\
\hline Preparatoria & \multicolumn{2}{|c|}{2} & \multicolumn{2}{|c|}{0} & \\
\hline Técnico profesional & \multicolumn{2}{|c|}{0} & \multicolumn{2}{|c|}{2} & \\
\hline Licenciatura & \multicolumn{2}{|c|}{11} & \multicolumn{2}{|c|}{8} & \\
\hline
\end{tabular}

Nota: + U Mann-Whitney; ++ Chi cuadrada.

Se realizó asimismo una comparación de las puntuaciones reportadas sobre el uso de las distintas estrategias de afrontamiento del estrés por ambos grupos. Únicamente se encontró una diferencia esta- dísticamente significativa en la relativa a la expresión emocional, como puede verse en la Tabla 2. En efecto, los pacientes sin RD utilizaban más dicha estrategia que los pacientes con ella.

Tabla 2. Diferencias intergrupales para las subescalas de afrontamiento.

\begin{tabular}{|l|r|r|r|r|r|}
\hline \multirow{2}{*}{ Estrategia de afrontamiento } & \multicolumn{2}{|c|}{$\begin{array}{c}\text { Sin RD } \\
\text { n= 21 }\end{array}$} & \multicolumn{2}{c|}{$\begin{array}{c}\text { Con RD } \\
\text { n= 21 }\end{array}$} & \multirow{2}{*}{$\boldsymbol{p}$} \\
\cline { 2 - 5 } & \multicolumn{1}{|c|}{ M } & \multicolumn{1}{|c|}{ D.E. } & \multicolumn{1}{c|}{ M } & \multicolumn{1}{c|}{ D.E. } & \\
\hline Resolución de problemas & 13.6 & 6.4 & 13.4 & 3.7 & .88 \\
\hline Reestructuración cognitiva & 14.4 & 5.3 & 13.3 & 4.1 & .47 \\
\hline Apoyo social & 12.1 & 5.5 & 11.4 & 5.3 & .67 \\
\hline Expresión emocional & 13.4 & 5.0 & 9.9 & 5.3 & .03 \\
\hline Evitación de problemas & 8.4 & 5.2 & 6.7 & 5.3 & .31 \\
\hline Pensamiento desiderativo & 13.5 & 5.2 & 13.6 & 5.3 & .93 \\
\hline Retirada social & 7.0 & 5.5 & 5.4 & 4.5 & .29 \\
\hline Autocrítica & 5.4 & 5.7 & 6.6 & 6.4 & .54 \\
\hline
\end{tabular}




\section{DISCUSIÓN}

Los resultados de esta investigación hecha en una población de pacientes con diagnóstico médico de diabetes mellitus tipo 2 indican que, al controlar el efecto de las variables sociodemográficas relativas al sexo, edad, estado civil, escolaridad y tiempo de padecer la enfermedad, la estrategia de afrontamiento ante el estrés denominada "expresión emocional" tuvo una frecuencia estadísticamente mayor $(p=.03)$ en pacientes sin retinopatía diabética que en quienes la padecían.

Pese a que Jaser, Patel, $\mathrm{Xu}$, Tamborlane y Grey (2017) afirman que el afrontamiento ante el estrés no está relacionado con la evolución de la diabetes mellitus tipo 2, los resultados de este trabajo coinciden con los de otros estudios que muestran los beneficios del afrontamiento pasivo (incluyendo la expresión emocional) en la población de pacientes. Por ejemplo, se ha hallado que esta estrategia de afrontamiento se asocia con una mayor estabilidad de los niveles de glucosa en sangre (Sürücü, Besen, Duman y Yeter, 2018), menos problemas emocionales (Yazla et al., 2018), percepción mayor de apoyo social (Ramkisson, Pillay y Sibanda, 2017) y mejor adaptación a los cambios en el tratamiento farmacológico (Kokoszka, 2017).

Particularmente, debe destacarse que si el afrontamiento pasivo, mediante el cual la persona regula cognitiva y emocionalmente lo que le acontece (Lazarus y Folkman, 1991), estabiliza los niveles de glucosa en la sangre (Sürücü et al., 2018), podría suponerse que tiene una probabilidad menor de desarrollar los efectos de la enfermedad al controlar los índices glucémicos.

Una posible explicación de estos hallazgos consiste en la posibilidad de que la expresión de las emociones negativas alivie su intensidad y, por lo tanto, disminuya el estrés. El hecho de que los pacientes manifiesten menos problemas emocionales (Yazla et al., 2018) puede sugerir que hacen un empleo más frecuente de estrategias de afrontamiento para liberar las emociones y disminuir así el estrés que ciertas situaciones les provocan (Cano et al., 2007).

Por otra parte, está comprobado que cuando los individuos inhiben activamente la expresión emocional, tienen un mayor riesgo de experimentar problemas de salud asociados a una respuesta fisiológica desregulada (Berry y Pennebaker, 1993). En el caso del presente estudio, la inhibición de las emociones está asociada a la retinopatía diabética, una complicación de la diabetes mellitus tipo 2, probablemente porque mantener el estrés en niveles altos provoca índices glucémicos elevados y, por ende, una mayor cantidad de enfermedades secundarias a este padecimiento.

Entre las limitaciones del presente estudio se encuentra el empleo de una muestra no representativa y conformada por conveniencia, y no haber incluido diversos factores, tales como el nivel glucémico de los participantes, la frecuencia e intensidad de los estresores y la adherencia al tratamiento, entre otros de índole psicosocial. Dado lo anterior, se sugiere que en futuros estudios se incluya la evaluación de la influencia de tales aspectos.

El hallazgo que relaciona una estrategia de afrontamiento con la complicación médica de una enfermedad crónico-degenerativa es pionero en el problema abordado; de hecho, tras llevar a cabo una extensa revisión bibliográfica no se encontraron estudios al respecto, por lo que constituye la base de futuros análisis a través de los cuales se pueda llevar a cabo la exploración de diversas variables psicológicas en pacientes con diabetes mellitus tipo 2 y distintas comorbilidades, a fin de comprender los mecanismos psicológicos de ajuste al proceso de la enfermedad, lo que podría traducirse en intervenciones efectivas, basadas en evidencia, que promuevan el bienestar subjetivo y físico de dicha población. En tal sentido, podrían considerarse los resultados de este trabajo para que la intervención médica integral incluya aspectos psicológicos en los que los pacientes con DM2 expresen sus emociones a través del diálogo o de actividades artísticas. 


\section{REFERENCIAS}

Azzollini, S., Bail, V., Vidal, V., Benvenuto, A. y Ferrer, L. (2015). Diabetes tipo 2: Depresión, ansiedad y su relación con las estrategias de afrontamiento utilizadas para la adhesión al tratamiento. Anuario de Investigaciones, 22, 287-291.

Berry, D.S. y Pennebaker, J.W. (1993). Nonverbal and verbal emotional expression and health. Psychotherapy and Psychosomatics, 59(1), 11-19.

Cano, F.J., Rodríguez, L. y García, J. (2007). Adaptación española del Inventario de Estrategias de Afrontamiento. Actas Espanolas de Psiquiatría, 35(1), 29-39.

Covarrubias, T., Delgado, I., Rojas, D. y Coria, M. (2017). Tamizaje en el diagnóstico y prevalencia de retinopatía diabética en atención primaria. Revista Médica de Chile, 145(5), 564-571.

Escobar M., C., Petrásovits, A., Peruga, A., Silva, N., Vives, M. y Robles, S. (2000). Mitos sobre la prevención y el control de las enfermedades no transmisibles en América Latina. Salud Pública de México, 42(1), 56-64.

Fernandez L., C., Martinez M., A., Martinez R., A., Benedi, J.M. y Traver, V. (2015). Diabetes care related process modelling using process mining techniques. Lessons learned in the application of interactive pattern recognition: Coping with the spaghetti effect. Proceedings of the Annual International Conference of the IEEE Engineering in Medicine and Biology Society, 2127-2130. doi: 10.1109/EMBC.2015.73188009.

García, J.C., Rodríguez M., D.L., Rentería, A., Rodríguez N., Y. y Melchor, J. (2016). Algunas reflexiones acerca de las aportaciones psicológicas en el control y regulación de la diabetes mellitus tipo 2. Revista Electrónica de Psicología Iztacala, $19,622-639$.

Gutiérrez J., P., Rivera D., J., Shamah L., T., Villalpando H., S., Franco, A., Cuevas N., L., Romero M., M. y Hernández Á., M. (2012). Encuesta Nacional de Salud y Nutrición 2012. Resultados Nacionales. Cuernavaca (México): Instituto Nacional de Salud Pública.

Hara, Y., Hisatomi, M., Ito, H., Nakao, M., Tsuboi, K. y Ishihara, Y. (2014). Effects of gender, age, family support, and treatment on perceived stress and coping of patients with type 2 diabetes mellitus. Biopsychosocial Medicine, 15, 8-16. doi: 10.1186/1751-0759-8-16.

Huang, C.Y., Lai, H.L., Lu, Y.C., Chen, W.K., Chi, S.C., Lu, C.Y. y Chen, C.I. (2016). Risk factors and coping style affect health outcomes in adults with type 2 diabetes. Biology Research for Nursing, 18, 82-89. doi: 10.1177/1099800415569845.

Jaser, S.S., Patel, N., Xu, M., Tamborlane, W.V. y Grey, M. (2017). Stress and coping predicts adjustment and glycemic control in adolescents with type 1 diabetes. Annals of Behavioral Medicine, 51(1), 30-38. doi: 10.1007/s12160-016-9825-5.

Kokoszka, A. (2017). Treatment adherence in patients with type 2 diabetes mellitus: correlates with different coping styles, low perception of self-influence on disease, and depressive symptoms. Patient Preference and Adherence, 11, 587-595. doi: 10.2147/PPA.S124605.

Lazarus, R.S. y Folkman, S. (1984). Stress, appraisal and coping. New York: Springer.

Lazarus, R.S. y Folkman, S. (1991). Estrés y procesos cognitivos. Barcelona: Martínez Roca.

Nishio, I. y Chujo, M. (2017). Self-stigma of patients with type 1 diabetes and their coping strategies. Yonago Acta Medica, 60(3), $167-173$.

Quiroz, C., Méndez, P., Valero, C.Z. y Trujano, R. (2010). Inventario de Estrategias de Afrontamiento: una replicación. Psicología y Salud, 20(2), 213-220.

Ramkisson, S., Pillay, B.J. y Sibanda, W. (2017). Social support and coping in adults with type 2 diabetes. African Journal of Primary Health Care and Family Medicine, 9(1), s.p. doi: 10.4102/phcfm.v9i1.1405.

Rodríguez, B. (2015). Prevención de ceguera por retinopatía diabética: ¿Dónde estamos? Revista Cubana de Oftalmología, 28(1), 119-128.

Romero, I., Dos Santos, M., Martins, T. y Zanetti, M. (2010). Autocuidado de personas con diabetes mellitus atendidas en un servicio de urgencia en México. Revista Latinoamericana de Enfermagem, 18(6), 1-9.

Sürücü, H.A., Besen, D.B., Duman, M. y Yeter, E. (2018). Coping with stress among pregnant women with gestational diabetes mellitus. Journal of Caring Sciences, 7(1), 9-15. doi: 10.15171/jcs.2018.002.

Yazla, E., Karadere, M.E., Küçükler, F.K., Karşıdağ, Ç., İnanç, L., Kankoç, E., Dönertaş, M. y Demir, E. (2018). The effect of religious belief and forgiveness on coping with diabetes. Journal of Religion and Health, 57, 1010-1019. doi: 10.1007/ s10943-017-0504-z. 